\title{
Approximate Partial Noether Operators of the Schwarzschild Spacetime
}

Ibrar Hussain, F. M. Mahomed, Asghar Qadir

To cite this article: Ibrar Hussain, F. M. Mahomed, Asghar Qadir (2010) Approximate Partial Noether Operators of the Schwarzschild Spacetime, Journal of Nonlinear Mathematical Physics 17:1, 13-25, DOI: https://doi.org/10.1142/S1402925110000556

To link to this article: https://doi.org/10.1142/\$1402925110000556

Published online: 04 January 2021 


\title{
APPROXIMATE PARTIAL NOETHER OPERATORS OF THE SCHWARZSCHILD SPACETIME
}

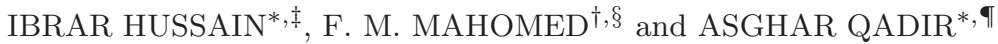 \\ ${ }^{*}$ Centre for Advanced Mathematics and Physics \\ National University of Sciences and Technology \\ Campus of the College of Electrical and Mechanical Engineering \\ Peshawar Road, Rawalpindi, Pakistan \\ ${ }^{\dagger}$ Centre for Differential Equations, Continuum Mechanics and Applications \\ School of Computational and Applied Mathematics \\ University of the Witwatersrand \\ Wits 2050, South Africa \\ $\ddagger_{\text {ihussain@camp.nust.edu.pk }}$ \\ §Fazal.Mahomed@wits.ac.za \\ \aqadirmath@yahoo.com
}

Received 13 July 2008

Accepted 11 June 2009

\begin{abstract}
The objective of this paper is twofold: (a) to find a natural example of a perturbed Lagrangian that has different partial Noether operators with symmetries different from those of the underlying Lagrangian. First we regard the Schwarzschild spacetime as a perturbation of the Minkowski spacetime and investigate the approximate partial Noether operators for this perturbed spacetime. It is shown that the Minkowski spacetime has 12 partial Noether operators, 10 of which are different from the 17 Noether symmetries for this spacetime. It is found that for the perturbed Schwarzschild spacetime we recover the exact partial Noether operators as trivial first-order approximate partial Noether operators and there is no non-trivial approximate partial Noether operator as for the Noether case. As a consequence we state a conjecture. (b) Then we prove a conjecture that the approximate symmetries of a perturbed Lagrangian form a subalgebra of the approximate symmetries of the corresponding perturbed Euler-Lagrange equations and illustrate it by our examples. This is in contrast to approximate partial Noether operators.
\end{abstract}

Keywords: Approximate partial Noether operators; Schwarzschild spacetime; perturbed Lagrangian.

\section{Introduction}

Differential equations (DEs) that involve some small perturbation often arise in mathematical physics. There are different computational techniques available for finding the approximate solutions of such equations. Baikov et al. [1] extended the Lie symmetry methods for DEs to the perturbed DEs for obtaining the approximate solutions of such perturbed DEs.

\footnotetext{
${ }^{\ddagger}$ Corresponding author.
} 
Noether's theorem [2] provides a relation between symmetries and conservation laws for Euler-Lagrange equations. The knowledge of Noether symmetries enables one to find first integrals of the corresponding Euler-Lagrange equations, for which methods are available in the literature (see e.g. [3]). These first integrals are of great importance from both the mathematical and physical points of view [4]. By the use of these first integrals one can reduce the order of the DEs (see e.g. [5]). Following Baikov's et al. idea Govinder et al. [6] presented an approximate version of the Noether theorem. These approximate Noether symmetries can be utilized to find approximate first integrals of the corresponding approximate (perturbed) Euler-Lagrange equations.

Noether's theorem (exact as well as approximate) needs the existence of a Lagrangian and that the corresponding Noether symmetry generators leave the action integral invariant. There are equations, e.g. scalar evolution equations (see e.g. [7]), which do not admit Lagrangian formulations. Other approaches do not assume the existence of a Lagrangian to construct first integrals or conservation laws for such equations [8-11]. One such approach proposed a method for constructing conservation laws via operators that are not necessarily symmetries of the underlying system of DEs. They call such operators partial Noether operators or Noether-type symmetries. For more general considerations we refer the reader to [12]. There are also equations or systems of equations with a small parameter, e.g. a system of two coupled van der Pol oscillators with linear diffusive coupling (see e.g. [13]) which do not admit a Lagrangian formulation in general. To construct first integrals for such perturbed equations without a Lagrangian formulation Johnpillai et al. developed the approximate partial Noether operators or approximate Noether-type symmetries [14]. Following this idea we investigate the approximate partial Noether-type symmetries of the perturbed Schwarzschild spacetime which is taken as a perturbation of the Minkowski spacetime. The approximate symmetries of the perturbed Schwarzschild spacetime were investigated in [15]. There was no non-trivial approximate Noether symmetry found for the perturbed Schwarzschild spacetime. Only the exact Noether symmetries were recovered as first-order approximate symmetries. In fact, a Lagrangian exists for the perturbed Schwarzschild spacetime. However we want to see the effectiveness of the approximate partial Noether-type symmetry approach in this article. In other words, as there is no non-trivial approximate Noether symmetry for the perturbed Schwarzschild spacetime, we want to see whether there exist non-trivial approximate partial Noether operators for this perturbed spacetime.

If a DE or system of DEs admits a Lagrangian formulation then the Noether symmetries and partial Noether-type symmetries are always the same and can be different only when the $\delta L / \delta x^{\mu}$ term appearing in the equations for calculating the partial Noethertype symmetries (given in the next section) involve the derivative which appears in the corresponding DEs [16]; this statement is also true for the approximate case [17]. We construct a partial Lagrangian for the Minkowski spacetime regarded as exact. This partial Lagrangian admits 12 partial Noether-type symmetries whereas the full Lagrangian admits 17 Noether symmetries [18]. The reason for this difference between Noether symmetries and partial Noether-type symmetries was explained earlier. Two of these 12 partial Noethertype symmetry generators are identical to those of the 17 Noether symmetry generators. Taking the small parameter $\epsilon$, (given by (43) in Sec. 3) we get a perturbed Schwarzschild spacetime [15]. For this perturbed spacetime there is no non-trivial approximate partial 
Noether-type symmetry and only the exact 12 partial Noether operators are recovered as trivial first-order approximate partial Noether-type symmetry generators. Some of these 12 partial Noether operators for the Minkowski spacetime form Abelian Lie algebras which are discussed in the last section.

In the second part of the paper we prove a conjecture, stated in [18], that the approximate Noether symmetries form a subalgebra of the approximate symmetries of the corresponding perturbed Euler-Lagrange equations. The corresponding statement is always true in the case of exact (unperturbed) symmetries [5]. Here we state and prove this as a theorem for the approximate case and provide two examples. In the case of perturbed partial EulerLagrange equations this is not true [14].

The plan of the paper is as follows. In the next section we briefly review the definitions of approximate Lie symmetries of DEs, Noether symmetries, approximate Noether symmetries, partial Noether operators and approximate partial Noether operators. In Sec. 3, partial Noether operators for the Minkowski spacetime and approximate partial Noether operators of the approximate Schwarzschild spacetime are given. In Sec. 4 we discuss the algebra of the approximate symmetries for the perturbed Lagrangians and of the corresponding perturbed Euler-Lagrange equations where we also give two examples. Finally a summary and discussion are given in Sec. 5 where we state an important conjecture.

\section{Preliminaries}

The first-order approximate Lie symmetry [1] of a perturbed system of ordinary differential equations (ODEs)

$$
\mathbf{E}=\mathbf{E}_{0}+\epsilon \mathbf{E}_{1}+O\left(\epsilon^{2}\right)
$$

is given by the generator

$$
\mathbf{X}=\mathbf{X}_{0}+\epsilon \mathbf{X}_{1}+O\left(\epsilon^{2}\right)
$$

where

$$
\mathbf{X}_{j}=\xi_{j}\left(s, x^{\mu}\right) \frac{\partial}{\partial s}+\eta_{j}^{\mu}\left(s, x^{\mu}\right) \frac{\partial}{\partial x^{\mu}}, \quad(j=0,1, \mu=0,1,2,3)
$$

if the symmetry condition

$$
\mathbf{X E}:=\left[\left(\mathbf{X}_{0}+\epsilon \mathbf{X}_{1}\right)\left(\mathbf{E}_{0}+\epsilon \mathbf{E}_{1}\right)\right]_{(1)}=O\left(\epsilon^{2}\right),
$$

is satisfied. Here $\mathbf{E}_{0}$ is the exact system of equations, $\mathbf{E}_{1}$ is the first-order approximate part of the perturbed system; $\mathbf{X}_{0}$ is the exact symmetry generator, $\mathbf{X}_{1}$ the first-order approximate part of the perturbed symmetry generator. These approximate symmetries do not necessarily form a Lie algebra but do form an "approximate Lie algebra" [19]. The perturbed system (1) always has the approximate symmetry generator $\epsilon \mathbf{X}_{0}$, which is a trivial approximate symmetry and $\mathbf{X}$ given by (2) with $\mathbf{X}_{0} \neq 0$ is a non-trivial (stable) approximate symmetry. 
Now we recall the definitions of Noether and first-order approximate Noether symmetries $[5,6]$. For a first-order perturbed system of ODEs (Euler-Lagrange equations)

$$
\mathbf{E}=\mathbf{E}_{0}+\epsilon \mathbf{E}_{1}=O\left(\epsilon^{2}\right),
$$

corresponding to a first-order Lagrangian, which is perturbed up to first-order in $\epsilon$,

$$
L\left(s, x^{\mu}, \dot{x}^{\mu}, \epsilon\right)=L_{0}\left(s, x^{\mu}, \dot{x}^{\mu}\right)+\epsilon L_{1}\left(s, x^{\mu}, \dot{x}^{\mu}\right)+O\left(\epsilon^{2}\right),
$$

the functional $\int_{V} L d s$ is invariant under the one-parameter group of transformations with approximate Lie symmetry generator (2), up to gauge

$$
A=A_{0}+\epsilon A_{1}
$$

and the first prolongation of $(3)$ is

$$
\mathbf{X}^{[1]}=\mathbf{X}+\left(\eta_{, s}^{\nu}+\eta_{, \mu}^{\nu} \dot{x}^{\mu}-\xi_{, s} \dot{x}^{\nu}-\xi_{, \mu} \dot{x}^{\mu} \dot{x}^{\nu}\right) \frac{\partial}{\partial \dot{x}^{\nu}}, \quad(\nu=0,1,2,3) .
$$

If

$$
\mathbf{X}_{0}^{[1]} L_{0}+\left(D_{s} \xi_{0}\right) L_{0}=D_{s} A_{0}
$$

and

$$
\mathbf{X}_{1}^{[1]} L_{0}+\mathbf{X}_{0}^{[1]} L_{1}+\left(D_{s} \xi_{1}\right) L_{0}+\left(D_{s} \xi_{0}\right) L_{1}=D_{s} A_{1}
$$

where $D_{s}$ is the total derivative operator given by

$$
D_{s}=\frac{\partial}{\partial s}+\dot{x}^{\mu} \frac{\partial}{\partial x^{i}}
$$

Here $L_{0}$ is the exact Lagrangian corresponding to the exact equations $\mathbf{E}_{0}=0$, and $L_{0}+\epsilon L_{1}$ the first-order approximate Lagrangian corresponding to the first-order perturbed equations $\mathbf{E}_{0}+\epsilon \mathbf{E}_{1}=0$. The definition of trivial and non-trivial approximate symmetries given above in the case of approximate symmetries of DEs, also holds for the approximate Noether symmetries. Like approximate symmetries of DEs, approximate Noether symmetries also form an approximate Lie algebra. The first-order approximate first integrals are defined by $I=I_{0}+\epsilon I_{1}$, where $I_{0}$ is the zeroth (exact part) and $I_{1}$ is the first-order approximate part of the first-order approximate first integrals

$$
\begin{aligned}
& I_{0}=\xi_{0} L_{0}+\left(\eta_{0}^{\mu}-\dot{x}^{\mu} \xi_{0}\right) \frac{\partial L_{0}}{\partial \dot{x}^{\mu}}-A_{0} \\
& I_{1}=\xi_{0} L_{1}+\xi_{1} L_{0}+\left(\eta_{0}^{\mu}-\dot{x}^{\mu} \xi_{0}\right) \frac{\partial L_{1}}{\partial \dot{x}^{\mu}}+\left(\eta_{1}^{\mu}-\dot{x}^{\mu} \xi_{1}\right) \frac{\partial L_{0}}{\partial \dot{x}^{\mu}}-A_{1} .
\end{aligned}
$$

If $I_{0}$ vanishes, then $I$ is an unstable approximate first integral and otherwise it is stable. A detailed discussion on the approximate first integrals for Hamiltonian dynamical system is given in [20]. Throughout this paper "." denotes differentiation with respect to the geodetic parameter $s$. 
Here we recall the definition of partial Lagrangians [12]. Consider an $k$ th-order DE system

$$
E_{\lambda}\left(s, x, \dot{x}^{\mu}, \ldots,\left(x^{\mu}\right)^{k}\right)=0, \quad \lambda=1, \ldots, m .
$$

Suppose that (14) are written as

$$
E_{\lambda}=E_{j}^{0}+E_{\lambda}^{1}, \quad \lambda=1, \ldots, m .
$$

If there exists a functional $L=L\left(s, x^{\mu}, \dot{x}^{\mu}, \ldots,\left(x^{\mu}\right)^{l}\right), l \leq k$ and nonzero differentiable function $f_{\mu}^{\lambda}$ such that (15) can be written as $\delta L / \delta \dot{x}^{\mu}=f_{\mu}^{\lambda} E_{\lambda}^{1}$ then, provided $E_{\alpha}^{1}$ nonzero, $L$ is called a partial Lagrangian of (15) otherwise it is the standard Lagrangian. The DEs of the form

$$
\frac{\delta L}{\delta x^{\mu}}=f_{\mu}^{\lambda} E_{\lambda}^{1}
$$

are called partial Euler-Lagrange equations.

Noether-type, or partial Noether, operators are defined as follows [12]. Consider a vector field given by (3) and its first prolongation (8). The vector field $\mathbf{X}$ is said to be a partial Noether operator corresponding to a partial Lagrangian $L\left(s, x^{\mu}, \dot{x}^{\mu}\right)$ of the partial EulerLagrange equations

$$
\ddot{x}^{\mu}=g\left(s, x^{\mu}, \dot{x}^{\mu}\right),
$$

if it can be calculated from

$$
\mathbf{X}^{[1]} L+\left(D_{s} \xi\right) L=\left(\eta^{\mu}-\xi \dot{x}^{\mu}\right) \frac{\delta L}{\delta x^{\mu}}+D_{s} A,
$$

where $A$ is known as the gauge function. These partial Noether-type symmetry generators do not form a Lie algebra in general. They only form a Lie algebra if these partial Noethertype symmetries coincide with the Noether symmetries, in which case the condition is stated in the beginning of the third paragraph of the first section.

Analogously to the Noether theorem one can state a Partial Noether, or Noether-like, theorem [16]:

Theorem 1. If $\boldsymbol{X}$ is a partial Noether operator corresponding to a partial Lagrangian $L\left(s, x^{\mu}, \dot{x}^{\mu}\right)$ of the partial Euler-Lagrange equations (17) then

$$
I=\xi L+\left(\eta^{\mu}-\dot{x}^{\mu} \xi\right) \frac{\partial L}{\partial \dot{x}^{\mu}}-A,
$$

is a first integral of (17) associated with $\boldsymbol{X}$.

Replacing $\mathbf{X}$ by $\mathbf{X}_{0}+\epsilon \mathbf{X}_{1}, \xi$ by $\xi_{0}+\epsilon \xi_{1}, \eta^{\mu}$ by $\eta_{0}^{\mu}+\epsilon \eta_{1}^{\mu}, L$ by $L_{0}+\epsilon L_{1}$ and $A$ by $A_{0}+\epsilon A_{1}$ in the definition of partial Noether operators and then comparing the coefficients of $\epsilon$ on both sides of the equations will give us the definition of the first-order approximate partial Noether operators $[14,17]$. The Noether-like theorem given above, and also the definition of trivial and non-trivial approximate symmetries given in the case of approximate symmetries of DEs, hold in the case of approximate partial Noether operators as well. In general these approximate partial Noether operators do not form an approximate Lie algebra. They only form an approximate Lie algebra under the condition stated in the beginning of the third paragraph of Sec. 1. 


\section{Approximate Partial Noether Operators for the Schwarzschild Spacetime}

We first give the partial Noether operators of the exact case, i.e. of Minkowski spacetime. The line element in spherical coordinates of the Minkowski spacetime is given by

$$
d s^{2}=d t^{2}-d r^{2}-r^{2}\left(d \theta^{2}+\sin ^{2} \theta d \phi^{2}\right) .
$$

This is a maximally symmetric spacetime having 10 Killing Vectors (KVs) which form the Poincaré symmetry algebra $s o(1,3) \oplus_{s} \mathbb{R}^{4}$ (where $\oplus_{s}$ denotes semi direct sum) [21]. A Lagrangian

$$
L=\dot{t}^{2}-\dot{r}^{2}-r^{2}\left(\dot{\theta}^{2}+\sin ^{2} \theta \dot{\phi}^{2}\right)
$$

for this spacetime admits a 17 dimensional Lie algebra which properly contain the 10 dimensional Poincaré symmetry algebra [18]. The geodesic equations for this spacetime are

$$
\begin{aligned}
\ddot{t} & =0, \\
\ddot{r}-r\left(\dot{\theta}^{2}+\sin ^{2} \theta \dot{\phi}^{2}\right) & =0, \\
\ddot{\theta}+\frac{2}{r} \dot{r} \dot{\theta}-\sin \theta \cos \theta \dot{\phi}^{2} & =0, \\
\ddot{\phi}+\frac{2}{r} \dot{r} \dot{\phi}+2 \cot \theta \dot{\theta} \dot{\phi} & =0 .
\end{aligned}
$$

From these equations we can write a partial Lagrangian (taken to be for a free particle) for this spacetime

$$
L_{p}=\frac{1}{2}\left[\dot{t}^{2}+\dot{r}^{2}+\alpha^{2}\left(\dot{\theta}^{2}+\dot{\phi}^{2}\right)\right]
$$

where $\alpha$ is a constant having dimensions of $r$. Using this partial Lagrangian in (18) and solving the resulting system of determining equations the following partial Noether generators are obtained

$$
\begin{aligned}
& \mathbf{X}_{0}=\frac{\partial}{\partial t}, \quad \mathbf{X}_{1}=\cos \theta \frac{\partial}{\partial r}-\frac{r}{\alpha^{2}} \sin \theta \frac{\partial}{\partial \theta} \\
& \mathbf{X}_{2}=r \cos \theta \frac{\partial}{\partial t}-t \cos \theta \frac{\partial}{\partial r}+\frac{r t}{\alpha^{2}} \sin \theta \frac{\partial}{\partial \theta}, \\
& \mathbf{X}_{3}=r \sin \theta \sin \phi \frac{\partial}{\partial t}-t \sin \theta \sin \phi \frac{\partial}{\partial r}-\frac{r t}{\alpha^{2}} \cos \theta \sin \phi \frac{\partial}{\partial \theta}-\frac{r t}{\alpha^{2}} \sin \theta \cos \phi \frac{\partial}{\partial \phi} \\
& \mathbf{X}_{4}=r \sin \theta \cos \phi \frac{\partial}{\partial t}-t \sin \theta \cos \phi \frac{\partial}{\partial r}-\frac{r t}{\alpha^{2}} \cos \theta \cos \phi \frac{\partial}{\partial \theta}+\frac{r t}{\alpha^{2}} \sin \theta \sin \phi \frac{\partial}{\partial \phi} \\
& \mathbf{X}_{5}=\sin \theta \sin \phi \frac{\partial}{\partial r}+\frac{r}{\alpha^{2}} \cos \theta \sin \phi \frac{\partial}{\partial \theta}+\frac{r}{\alpha^{2}} \sin \theta \cos \phi \frac{\partial}{\partial \phi} \\
& \mathbf{X}_{6}=\sin \theta \cos \phi \frac{\partial}{\partial r}+\frac{r}{\alpha^{2}} \cos \theta \cos \phi \frac{\partial}{\partial \theta}-\frac{r}{\alpha^{2}} \sin \theta \sin \phi \frac{\partial}{\partial \phi} \\
& \mathbf{X}_{7}=\frac{r^{2}}{\alpha^{2}} \sin { }^{2} \theta \frac{\partial}{\partial \phi}, \\
& \mathbf{X}_{8}=s \mathbf{X}_{0}, \quad \mathbf{X}_{9}=s \mathbf{X}_{1}, \quad \mathbf{X}_{10}=s \mathbf{X}_{5}, \quad \mathbf{X}_{11}=s \mathbf{X}_{6},
\end{aligned}
$$


and the gauge function is

$$
A=a_{0} t+a_{1}+r\left[a_{2} \cos \theta+\sin \theta\left(a_{5} \sin \phi+a_{6} \cos \phi\right)\right]
$$

where $a_{0}, a_{1}, a_{2}, a_{5}$ and $a_{6}$ are arbitrary constants of integration. The operators $\mathbf{X}_{0}$ and $\mathbf{X}_{8}$ given in (27) and (34) which form an Abelian Lie algebra are also the Noether symmetry generators of the Minkowski spacetime. The other 10 generators given in (27)-(34) are different from the Noether symmetry generators for the Minkowski spacetime.

The Schwarzschild spacetime is a spherically symmetric, static solution of the Einstein vacuum field equations. The line element for this spacetime is given by [22]

$$
d s^{2}=\left(1-\frac{2 G m}{r c^{2}}\right) d t^{2}-\left(1-\frac{2 G m}{r c^{2}}\right)^{-1} d r^{2}-r^{2}\left(d \theta^{2}+\sin ^{2} \theta d \phi^{2}\right)
$$

where $m$ is the mass of the gravitating source. This metric reduces to the Minkowski metric when $m=0$. This spacetime has $4 \mathrm{KVs}$ which give the energy and angular momentum conservation laws [23].

For this spacetime a Lagrangian is given by

$$
L=\left(1-\frac{2 G m}{r c^{2}}\right) \dot{t}^{2}-\left(1-\frac{2 G m}{r c^{2}}\right)^{-1} \dot{r}^{2}-r^{2}\left(\dot{\theta}^{2}+\sin ^{2} \theta \dot{\phi}^{2}\right) .
$$

This Lagrangian admits a 5 dimensional Lie algebra i.e. $4 \mathrm{KVs}$ and $\partial / \partial s$ [18]. The approximate symmetries of this spacetime were considered in [15]. For this purpose this spacetime was taken as a perturbation of the Minkowski spacetime with the small parameter i.e.

$$
\epsilon=\frac{2 G m}{c^{2}}
$$

and it was shown that there is no non-trivial approximate Noether symmetry for this spacetime. Only the exact symmetries were recovered as first-order trivial approximate symmetries which yield approximate conservation laws for this spacetime.

Now for the approximate partial Noether operators of the perturbed Schwarzschild spacetime we also use the same $\epsilon$ given by (38). A first-order Lagrangian for the perturbed Schwarzschild spacetime is given by

$$
L=\dot{t}^{2}-\dot{r}^{2}-r^{2}\left(\dot{\theta}^{2}+\sin ^{2} \theta \dot{\phi}^{2}\right)-\epsilon \frac{1}{r}\left(\dot{t}^{2}+\dot{r}^{2}\right)+O\left(\epsilon^{2}\right) .
$$

When $\epsilon=0$ this Lagrangian reduces to that of the Minkowski spacetime. The geodesic equations for the Schwarzschild spacetime are given by

$$
\begin{aligned}
\ddot{t}+\nu^{\prime} \dot{t} \dot{r} & =0, \\
\ddot{r}+\frac{1}{2}\left(e^{\nu}\right)^{\prime}\left(e^{\nu} c^{2} \dot{t}^{2}-e^{-\nu} \dot{r}^{2}\right)-r e^{\nu}\left(\dot{\theta}^{2}+\sin ^{2} \theta \dot{\phi}^{2}\right) & =0, \\
\ddot{\theta}+\frac{2}{r} \dot{r} \dot{\theta}-\sin \theta \cos \theta \dot{\phi}^{2} & =0, \\
\ddot{\phi}+\frac{2}{r} \dot{r} \dot{\phi}+2 \cot \theta \dot{\theta} \dot{\phi} & =0,
\end{aligned}
$$


with $\nu=1-2 G m / r c^{2}$, which for the approximate case can be written as $\nu=1-\epsilon / r$. From these geodesic equations we have the same partial Lagrangian given by (26). We use this partial Lagrangian (26), the approximate geodesic equations given by (40)-(43) and the exact partial Noether generators given by (27)-(34) in the definition of approximate partial Noether operators. In the resulting system of determining equations 11 of the 12 exact partial Noether generators appear. But for the consistency of these determining equations all the 11 constants have to be eliminated and the system again becomes homogeneous. The resulting system is once more the same as for the Minkowski spacetime, yielding 12 first-order approximate partial Noether generators given by (27)-(34). Thus like the case of first-order approximate Noether symmetries for the perturbed Schwarzschild spacetime [15], there is no non-trivial first-order approximate partial Noether-type symmetry for this perturbed spacetime.

\section{Relationship of the Approximate Noether Symmetries with the Approximate Symmetries of the Corresponding Perturbed Euler-Lagrange Equations}

The set of symmetry generators of a Lagrangian is always contained in the set of the symmetry generators of the corresponding Euler-Lagrange equations or in other words the Noether symmetries always form a subalgebra of the Lie symmetries of the corresponding Euler-Lagrange equations [5]. However, this is not true for the approximate partial Noether operators. In [18] it was conjectured that the above mentioned result also holds for the case of approximate symmetries. Here we prove that conjecture as a theorem.

Theorem 2. The approximate Lie algebra of the approximate symmetries of a perturbed Lagrangian form an approximate subalgebra of the approximate symmetries of the corresponding perturbed Euler-Lagrange (geodesic) equations.

Proof. The proof of this theorem is similar to the proof for the exact case which is given in [5]. Replacing the exact quantities by the the perturbed one in the proof of the exact case, i.e. the exact Euler-Lagrange equations $\mathbf{E}$ by the perturbed Euler-Lagrange equations $\mathbf{E}_{0}+\epsilon \mathbf{E}_{1}+\cdots+\epsilon^{k} \mathbf{E}_{k}$ etc, one arrives at the proof of the above theorem (2).

Now we illustrate Theorem 2 by two examples.

Example 1. The orbital equation for the Schwarzschild spacetime is [15]

$$
\frac{d^{2} u}{d \phi^{2}}+u=\frac{G m}{h^{2}}+\frac{3 G m}{c^{2}} u^{2}
$$

where $h$ is the classical angular momentum per unit mass and $u=1 / r$. In the classical limit $c \rightarrow \infty$ it gives the classical orbital equation. Using the same $\epsilon$ given by (38) in (44) the first-order approximate orbital equation is obtained

$$
\frac{d^{2} u}{d \phi^{2}}+u=\frac{\epsilon}{2}\left(\frac{c^{2}}{h^{2}}+3 u^{2}\right) .
$$


Taking $\epsilon=0$ in (45), the exact symmetry generators are [24]

$$
\begin{aligned}
& \mathbf{X}_{0}=u \frac{\partial}{\partial u}, \quad \mathbf{X}_{1}=\cos \phi \frac{\partial}{\partial u}, \quad \mathbf{X}_{2}=\sin \phi \frac{\partial}{\partial u}, \quad \mathbf{X}_{3}=\frac{\partial}{\partial \phi}, \\
& \mathbf{X}_{4}=\cos 2 \phi \frac{\partial}{\partial \phi}-u \sin 2 \phi \frac{\partial}{\partial u}, \quad \mathbf{X}_{5}=\sin 2 \phi \frac{\partial}{\partial \phi}+u \cos 2 \phi \frac{\partial}{\partial u} \\
& \mathbf{X}_{6}=u \cos \phi \frac{\partial}{\partial \phi}-u^{2} \sin \phi \frac{\partial}{\partial u}, \quad \mathbf{X}_{7}=u \sin \phi \frac{\partial}{\partial \phi}+u^{2} \cos \phi \frac{\partial}{\partial u}
\end{aligned}
$$

The perturbed orbital equation (45) admits a 10 dimensional approximate Lie algebra, i.e. the 8 trivial first-order approximate symmetries and two non-trivial (stable) first-order approximate symmetries [15]

$$
\begin{aligned}
& \mathbf{X}_{a 1}=\sin \phi \frac{\partial}{\partial u}+\epsilon\left(2 \sin \phi \frac{\partial}{\partial \phi}+u \cos \phi \frac{\partial}{\partial u}\right) \\
& \mathbf{X}_{a 2}=\cos \phi \frac{\partial}{\partial u}-\epsilon\left(2 \cos \phi \frac{\partial}{\partial \phi}-u \sin \phi \frac{\partial}{\partial u}\right)
\end{aligned}
$$

A Lagrangian for the perturbed orbital equation (45) is

$$
L=\frac{1}{2}\left(u^{\prime 2}-u^{2}\right)+\frac{\epsilon}{2}\left(\frac{c^{2}}{h^{2}} u+u^{3}\right) .
$$

Taking $\epsilon=0$ in the above perturbed Lagrangian and using this exact Lagrangian in the definition of Noether symmetries we get the 5 symmetry generators $\mathbf{X}_{1}, \mathbf{X}_{2}, \mathbf{X}_{3}, \mathbf{X}_{4}$ and $\mathbf{X}_{5}$ given in (46) and (47) and the gauge function is

$$
A=-u^{2}\left(a_{1} \cos 2 \phi+a_{2} \sin 2 \phi\right)-u\left(a_{3} \sin \phi-a_{4} \cos \phi\right) .
$$

These symmetries of the exact (when $\epsilon=0$ ) Lagrangian form a 5 dimensional subalgebra of the 8 dimensional symmetry algebra of the exact orbital equation.

Now the perturbed Lagrangian (51) and the 5 exact Noether symmetry generators are used in the definition of approximate Noether symmetries. In the resulting system of equations 2 of the 5 exact symmetry constants appear. Integrating these equations we get the two non-trivial first-order approximate symmetry generators given by (49) and (50) along with the 5 trivial first-order approximate symmetry generators given in (46) and (47). The gauge function in this case is

$$
\begin{aligned}
A= & -u^{2}\left(b_{1} \cos 2 \phi+b_{2} \sin 2 \phi\right)+\frac{1}{2}\left(a_{0} \sin \phi-a_{1} \cos \phi\right)\left(\frac{c^{2}}{h^{2}}-u^{2}\right) \\
& -u\left(b_{3} \sin \phi-b_{4} \cos \phi\right)+b_{5} .
\end{aligned}
$$

These 7 first-order approximate symmetries of the perturbed Lagrangian (51) form a subalgebra of the 10 dimensional approximate symmetry algebra of the perturbed equation (45). The stable approximate first integrals corresponding to the stable approximate symmetry 
generators (49) and (50) are

$$
\begin{aligned}
& I_{a_{1}}=u^{\prime} \sin \phi-u \cos \phi-\epsilon\left[u^{\prime 2} \sin \phi-u u^{\prime} \cos \phi+\frac{1}{2}\left(u^{2}+\frac{c^{2}}{h^{2}}\right) \sin \phi\right] \\
& I_{a_{2}}=u^{\prime} \cos \phi+u \sin \phi+\epsilon\left[u^{\prime 2} \cos \phi+u u^{\prime} \sin \phi+\frac{1}{2}\left(u^{2}+\frac{c^{2}}{h^{2}}\right) \cos \phi\right] .
\end{aligned}
$$

Example 2. Consider the damped harmonic oscillator

$$
y^{\prime \prime}+y+\epsilon y^{\prime}=0
$$

This equation admits an 11 dimensional approximate Lie algebra with generators [25]

$$
\begin{aligned}
& \mathbf{X}_{0}= y \frac{\partial}{\partial y}, \quad \mathbf{X}_{1}=\cos x \frac{\partial}{\partial y}, \quad \mathbf{X}_{2}=\sin x \frac{\partial}{\partial y}, \quad \mathbf{X}_{3}=\frac{\partial}{\partial x} \\
& \mathbf{X}_{4}=\cos 2 x \frac{\partial}{\partial x}-y \sin 2 x \frac{\partial}{\partial y}, \quad \mathbf{X}_{5}=\sin 2 x \frac{\partial}{\partial x}+y \cos 2 x \frac{\partial}{\partial y} \\
& \mathbf{X}_{6}=y \cos x \frac{\partial}{\partial x}-y^{2} \sin x \frac{\partial}{\partial y}, \quad \mathbf{X}_{7}=y \sin x \frac{\partial}{\partial x}+y^{2} \cos x \frac{\partial}{\partial y} \\
& \mathbf{X}_{8}=\cos x \frac{\partial}{\partial y}-\epsilon x \sin x \frac{\partial}{\partial y}, \quad \mathbf{X}_{9}=\sin x \frac{\partial}{\partial y}-\epsilon x \sin x \frac{\partial}{\partial y} \\
& \mathbf{X}_{10}=y \cos x \frac{\partial}{\partial x}-y^{2} \sin x \frac{\partial}{\partial y}-\epsilon\left[\left(\frac{-5}{3} x y \cos x+4(\sin x-x \cos x)\right) \frac{\partial}{\partial x}\right. \\
&\left.+\left(\frac{-5}{3} y^{2}(\cos x-x \sin x)+2 y^{2} \cos x+2 x y \sin x\right) \frac{\partial}{\partial y}\right] .
\end{aligned}
$$

A Lagrangian for $(56)$ is [25]

$$
L=\frac{1}{2}\left(y^{\prime 2}-y^{2} \cot ^{2} x\right)+y y^{\prime} \cot x+\epsilon\left(y y^{\prime}+x y^{\prime 2}-2 x y y^{\prime} \cot x-y^{2} \cot x+x y^{2}\right) .
$$

This first-order perturbed Lagrangian (62) admit an 8 dimensional approximate Noether symmetry algebra with symmetry generators $\mathbf{X}_{1}, \mathbf{X}_{2}, \mathbf{X}_{3}, \mathbf{X}_{4}, \mathbf{X}_{5}, \mathbf{X}_{8}, \mathbf{X}_{9}$ and $\mathbf{X}_{10}$ given in (57), (58), (60) and (61). This 8 dimensional approximate symmetry algebra is a subalgebra of the above 11 dimensional approximate symmetry algebra of the perturbed equation (56). In this case the gauge function is zero.

Remark. Theorem 2 does not apply to partial Noether operators. As we have seen in the previous section, the 12 approximate partial Noether operators do not form an algebra.

\section{Summary and Discussion}

In this paper we first discussed approximate partial Noether operators of the (perturbed) Schwarzschild spacetime, taken as a perturbation of the Minkowski spacetime. Then we discussed the relationship between the approximate symmetry algebra of the perturbed Lagrangian and the corresponding perturbed Euler-Lagrange equations. We constructed a partial Lagrangian (26) for the Minkowski spacetime. A standard Lagrangian exists for this spacetime which has a 17 dimensional Lie algebra [18]. The partial Lagrangian (26) for this spacetime has only 12 generators, 2 of these are the same as the Noether symmetries for 
this spacetime and the remaining 10 are different. These 12 partial Noether operators given by (27)-(34) do not form a Lie algebra. But there are certain subsets of these 12 partial Noether operators that form 3 dimensional and 2 dimensional Abelian Lie algebras. The 3 dimensional Abelian Lie algebras are $\left\langle\mathbf{X}_{\mathbf{0}}, \mathbf{X}_{\mathbf{8}}, \mathbf{X}_{\mathbf{i}}\right\rangle$ and the 2 dimensional Abelian Lie algebras are $\left\langle\mathbf{X}_{\mathbf{0}}, \mathbf{X}_{\mathbf{8}}\right\rangle,\left\langle\mathbf{X}_{\mathbf{0}}, \mathbf{X}_{\mathbf{i}}\right\rangle$ and $\left\langle\mathbf{X}_{\mathbf{8}}, \mathbf{X}_{\mathbf{i}}\right\rangle$ where $i=1,5,6,7,9,10,11$. Each of these 2 dimensional Lie algebras is a subalgebra of the corresponding 3 dimensional Lie algebra.

The perturbed Schwarzschild spacetime admits a 17 dimensional trivial first-order approximate Noether symmetry algebra [18]. We wanted to see the effectiveness of the approximate partial Noether operators $[14,17]$ and to check whether there exists any nontrivial partial Noether operator for the perturbed Schwarzschild spacetime in contrast to the approximate Noether symmetries for this spacetime. For this purpose we perturbed the Minkowski spacetime with a small parameter $\epsilon$ given by (38) to get the perturbed Schwarzschild spacetime. This perturbed spacetime reduces to the Minkowski spacetime for $\epsilon=0$. The 12 exact partial Noether operators as trivial first-order approximate partial Noether operators are obtained for this perturbed spacetime. Hence as for the approximate Noether symmetries for this perturbed Schwarzschild spacetime, there is no non-trivial approximate partial Noether operator. As a consequence we state the following conjecture.

Conjecture. If there does not exist a non-trivial approximate Noether symmetry for a perturbed Lagrangian corresponding to the perturbed Euler-Lagrange equations, then there will be no non-trivial approximate partial Noether-type symmetry for the same case.

It would be of interest to investigate the approximate partial Noether operators of the examples discussed in Sec. 4, for which there exist non-trivial approximate Noether symmetries and to check whether there exist a non-trivial approximate partial Noether operator for these examples. A general proof will be more interesting.

A conjecture was stated in [18], i.e. the approximate symmetries of a perturbed Lagrangian form a subalgebra of the approximate symmetries of the corresponding perturbed Euler-Lagrange equations. Here this conjecture is proved as a theorem. The proof is straightforward and can be obtained from the proof for the exact case [5]. As an example we gave the orbital equation of the Schwarzschild spacetime [15]. This orbital equation has only one symmetry $\partial / \partial \phi$. We used the small parameter $\epsilon$ given by (38) to treat this equation as perturbed. In the exact case (when $\epsilon=0$ ) this equation admits 8 symmetry generators given by (46)-(48). A Lagrangian for this orbital equation admits 5 symmetry generators, given in (46) and (47), which form a subalgebra of the 8 dimensional algebra for the unperturbed orbital equation. The perturbed orbital equation (45) admits 2 non-trivial (stable) and 8 trivial first-order approximate symmetries [15]. The perturbed Lagrangian (51) admits a 7 dimensional approximate Lie algebra, 5 of these are trivial and 2 are non-trivial firstorder approximate symmetries. The 2 non-trivial first-order approximate Noether symmetries for this perturbed Lagrangian (51) are the same as those of the perturbed orbital equation (45). The stable first-order approximate first integrals corresponding to the 2 nontrivial approximate Noether symmetries (49) and (50) are given by (54) and (55). These 7 approximate Noether symmetries for the perturbed Lagrangian (51) form an approximate subalgebra of the 10 dimensional approximate symmetry algebra of the perturbed orbital equation (45). In the second example of the damped harmonic oscillator we also saw that 8 approximate Noether symmetry generators (3 non-trivial and 5 trivial) form a subalgebra 
of the 11 dimensional (3 non-trivial and 8 trivial) approximate Lie algebra. Hence, as in the exact (unperturbed) case, also in the perturbed case, approximate Noether symmetries always form an approximate subalgebra of the approximate Lie algebra of the approximate symmetries of the corresponding perturbed Euler-Lagrange equations. This is in contrast to approximate partial Noether operators $[14,17]$. This is also evident for the 12 partial Noether operators (27)-(34) of the Minkowski spacetime which do not form an algebra. In both the examples 1 and 2 it is seen that all the non-trivial approximate symmetries of the perturbed Euler-Lagrange equations are also the non-trivial approximate symmetries of the corresponding perturbed Lagrangians. A general proof of this or construction of a counter example is worth exploring.

In the first example the perturbed non-linear equation admits a 10 dimensional approximate symmetry algebra and the corresponding perturbed Lagrangian admits a 7 dimensional approximate symmetry algebra. While in the second example the perturbed linear equation admits a 11 dimensional approximate symmetry algebra and the corresponding perturbed Lagrangian admits 8 dimensional approximate symmetry algebra. A reason for this difference seems to be the non-linearity of the perturbed equation in the first example and the linearity of the perturbed equation in the second example. To find the maximum dimensions of the approximate algebra for the perturbed ODEs (linear and nonlinear) and Lagrangians is also worth exploring.

\section{Acknowledgments}

IH would like to thank Higher Education Commission of Pakistan (HEC) for their full financial support and DECMA of the University of Witwatersrand, Johannesburg, where the writing-up of this paper was completed.

\section{References}

[1] V. A. Baikov, R. K. Gazizov and N. H. Ibragimov, Math. USSR Sbornik 64 (1989) 427.

[2] E. Noether, Invariant variations problems, Nachr. Konig. Gissell. Wissen., Gottingen, Math.Phys. Kl. 2 (1918) 235. (English translation in transport theory and Statistical Physics 1 (1971)) 186.

[3] L. V. Ovsiannikov, Group Analysis of Differential Equations (Academic Press, New York, 1980).

[4] H. Goldstein, Classical Mechanics (Addison-Wesley, 1950).

[5] P. J. Olver, Applications of Lie Groups to Differential Equations (Springer-Verlag, New York, 1993).

[6] K. S. Govinder, T. G. Heil and T. Uzer, Phys. Lett. A A240 (1998) 127.

[7] I. M. Anderson and T. E. Duchamp, J. Diff. Equa. 51 (1984) 1.

[8] N. H. Ibragimov, CRC Handbook of Lie Group Analysis of Differential Equations, Vol. 1, ed. N. H. Ibragimov (CRC Press, Boca Raton, Florida, 1994).

[9] C. M. Khalique and F. M. Mahomed, Math. Probl. Engin. 26 (2005) 141.

[10] H. Steudel, Zeit Naturforsch 17A (1962) 129.

[11] S. C. Anco and G. W. Bluman, Euro. J. Appli. Math. 9 (2002) 567.

[12] A. H. Kara and F. M. Mahomed, Nonlinear Dyn. 45 (2006) 367.

[13] L. A. Low, P. G. Reinhall, D. W. Storti and E. B. Goldman, Struct. Control Health Monit. 13 (2006) 417.

[14] A. G. Johnpillai, A. H. Kara and F. M. Mahomed, J. Comp. Appl. Math. 223 (2009) 508.

[15] A. H. Kara, F. M. Mahomed and A. Qadir, Nonlinear Dyn. 51 (2008) 183.

[16] I. Naeem and F. M. Mahomed, J. Math. Anal. Appl. 342 (2008) 70. 
[17] I. Naeem and F. M. Mahomed, Nonlinear Dyn. (2009), DOI 10.1007/s11071-008-9441-4.

[18] I. Hussain, F. M. Mahomed and A. Qadir, Gen. Rel. Grav. (2009) DOI 10.1007/s10714-0090772-3.

[19] R. K. Gazizov, J. Nonlinear Math. Phys. 3 (1996) 96.

[20] G. Unal, Phys. Lett. A 269 (2000) 13.

[21] D. Kramer, H. Stephani, M. A. H. MacCullum and E. Herlt, Exact Solutions of Einstein Field Equations (Cambridge University Press, Cambridge, 1980).

[22] C. W. Misner, K. S. Thorne and J. A. Wheeler, Gravitation (W. H. Freeman and Company, San Francisco, 1973).

[23] G. S. Hall, Symmetries and Curvature Structure in General Relativity (World Scientific, Singapore, 2004).

[24] I. Hussain, F. M. Mahomed and A. Qadir, SIGMA 3 (2007) 115, arXiv.0712.1089.

[25] T. Feroze and A. H. Kara, Int. J. Non-linear Mech. 37 (2002) 275. 